\title{
Perturbing Open Cavities: Anomalous Resonance Frequency Shifts in a Hybrid Cavity-Nanoantenna System
}

\author{
Freek Ruesink, ${ }^{1}$ Hugo M. Doeleman, ${ }^{1,2}$ Ruud Hendrikx, ${ }^{1}$ A. Femius Koenderink, ${ }^{1,2}$ and Ewold Verhagen ${ }^{1, *}$ \\ ${ }^{1}$ FOM Institute AMOLF, Science Park 104, 1098 XG Amsterdam, The Netherlands \\ ${ }^{2}$ Institute of Physics, University of Amsterdam, P.O. Box 94485, 1090 GL Amsterdam, The Netherlands
}

(Received 10 August 2015; published 11 November 2015)

\begin{abstract}
The influence of a small perturbation on a cavity mode plays an important role in fields like optical sensing, cavity quantum electrodynamics, and cavity optomechanics. Typically, the resulting cavity frequency shift directly relates to the polarizability of the perturbation. Here, we demonstrate that particles perturbing a radiating cavity can induce strong frequency shifts that are opposite to, and even exceed, the effects based on the particles' polarizability. A full electrodynamic theory reveals that these anomalous results rely on a nontrivial phase relation between cavity and nanoparticle radiation, allowing backaction via the radiation continuum. In addition, an intuitive model based on coupled mode theory is presented that relates the phenomenon to retardation. Because of the ubiquity of dissipation, we expect these findings to benefit the understanding and engineering of a wide class of systems.
\end{abstract}

DOI: 10.1103/PhysRevLett.115.203904

PACS numbers: 42.82.Fv, 42.25.Fx, 42.25.Hz, 78.67.-n

The fact that a small perturbation of a potential can influence the distribution of a systems energy levels is a well-known principle permeating various branches of physics. In quantum mechanics, for example, the effect of a perturbing potential $H^{\prime}$ on an eigenstate $\left|\psi_{0}\right\rangle$ is that it modifies its unperturbed energy $U_{0}$ by an amount $\delta U=\left\langle\psi_{0}\left|H^{\prime}\right| \psi\right\rangle$, where $|\psi\rangle$ is the new eigenstate, and we assume $\left\langle\psi_{0} \mid \psi\right\rangle \approx 1$. In electrodynamics, a local change of potential (i.e., permittivity) can impact the frequency of a resonant cavity. This is at the basis of many applications that use the influence of a perturbing atom, molecule, or dielectric body to establish an interaction that can be exploited for optical sensing or control [1-8]. The shift of a mode's complex eigenfrequency $\omega=\omega_{\mathrm{c}}-\mathrm{i} \kappa / 2$, with cavity resonance frequency $\omega_{\mathrm{c}}$ and linewidth $\kappa$, due to a local permittivity perturbation $\Delta \epsilon$ contained in a volume $\Delta V$ is given by $\delta \omega / \omega$ $=-\int_{\Delta V} d V\left[\epsilon_{0} \Delta \epsilon \mathbf{E}_{0}^{*} \cdot \mathbf{E}_{p}\right] / 4 U_{0}$. Here, $\mathbf{E}_{0}$ and $U_{0}$ represent the field and total energy of the unperturbed cavity mode, respectively, and $\mathbf{E}_{p}$ is the perturbed field $[9,10]$. In particular, if $\Delta V$ is small enough such that the perturbing particle can be described in the dipole approximation, the complex frequency shift is directly related to the particle polarizability $\alpha$, reading $\delta \omega / \omega=-\alpha\left|\mathbf{E}_{0}\right|^{2} / 4 U_{0} \quad$ [9]. However, this widely employed result of Bethe-Schwinger perturbation theory [10] is strictly only valid when radiation to the far field is negligible. In several recent developments, radiation loss proved decisive in determining a system's eigenmode, e.g., for so-called "states bound in the continuum" [11-13] and in describing complex plasmonic resonators $[14,15]$. In that context, the question arises to what extent the conventional paradigm to determine perturbed cavity frequencies holds in practical, open, systems.

Here, we study the eigenfrequencies of a radiating optical cavity as it is perturbed by carefully designed resonant plasmonic nanoparticles. For a resonant perturbation with center frequency $\omega_{\mathrm{a}}$ and linewidth $\gamma$, the value of $\alpha$ strongly depends on the detuning $\Delta=\omega_{\mathrm{c}}-\omega_{\mathrm{a}}$. In absence of radiation, the cavity mode thus redshifts (blueshifts) at negative (positive) values of $\Delta$, and the linewidth broadens near $\Delta=0$, in direct response to $\operatorname{Re}[\alpha]$ and $\operatorname{Im}[\alpha]$, respectively, [Fig. 1(a)]. Tuning $\omega_{\mathrm{a}}$ by varying the length of the (plasmonic) resonators allows us to systematically study the induced cavity response as a function of detuning. Importantly, our experiment is designed such that radiation losses from the cavity mode and plasmonic resonators overlap. This, as we will show, leads to a strong additional contribution to $\delta \omega$ which is not captured by $\alpha$. We study this "radiation interaction", as we will call the effect, in detail and show that it can induce strong eigenfrequency shifts that are opposite to, and even exceed, the effects based on the particles' polarizability.

A sketch of the system is shown in Fig. 1(b). The experiments are performed using a fundamental cavity mode (194.4 THz, Q $6.5 \times 10^{6}$, TE polarized) of a toroidal silica microcavity fabricated on the edge of a silicon chip [ $\approx 36 \mu \mathrm{m}$ diameter, Fig. 1(c)] [16,17]. The cavity is perturbed by gold nanoantennas deposited on a glass substrate [Fig. 1(d)], which are controllably placed in the evanescent field of the cavity. The antennas (nanorods of length $L$, width $120 \mathrm{~nm}$ and thickness $40 \mathrm{~nm}$ ) are aligned with their (long) principal dipole axis to the polarization of the cavity mode. A frequency-swept narrow band laser source $(\sim 0.7 \mu \mathrm{W})$ is coupled into a tapered fiber that is brought close to the cavity. The transmission spectrum through the fiber shows a Lorentzian dip around the cavity resonance frequency [Fig. 1(e)], from which we determine the (perturbed) resonance frequency $\omega_{\mathrm{c}}$ and linewidth $\kappa$ of the cavity mode. Independent normal-incidence 
(a)
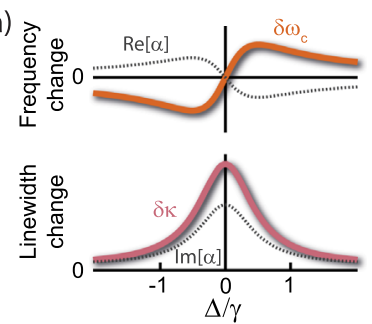

(c)

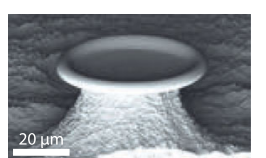

(e)

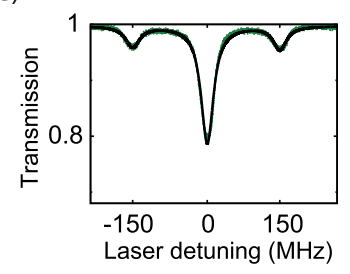

(b)

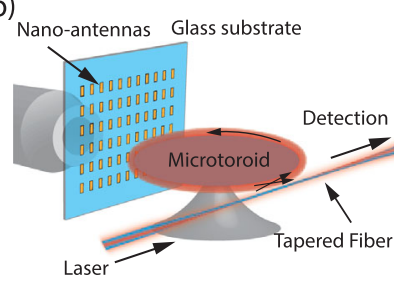

(d)

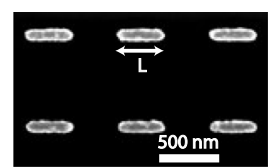

(f)

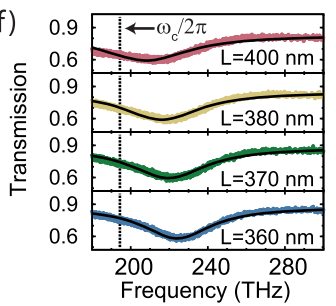

FIG. 1 (color online). (a) Cavity frequency and linewidth change due to perturbation by a small resonator, considering only the resonator polarizability. Dashed lines indicate the real (top) and imaginary (bottom) part of the polarizability as a function of $\Delta / \gamma$. (b) An array of perturbing gold nanoantennas is placed in the near field of a toroidal microcavity. Light transmission through the tapered fiber is detected to determine the cavity eigenfrequency shift. (c) Scanning electron microscopy (SEM) image of a high- $Q$ silica microtoroid at the edge of a chip. (d) SEM image of gold nanoantennas on a glass substrate. (e) Transmittance through the tapered fiber around a cavity mode resonance. A Lorentzian fit (black solid line) is used to determine the optical linewidth $(\approx 30 \mathrm{MHz})$ and frequency of the cavity. The sidebands result from a $150-\mathrm{MHz}$ phase modulation used to calibrate the frequency axis. (f) Normal-incidence transmission spectra of gold nanoantenna (design length $L$ ) arrays. Lorentzian fits (sold lines) give the antenna resonance frequency and linewidth. The dashed line indicates the cavity frequency.

transmission measurements [Fig. 1(f)] on the fabricated antenna arrays, in absence of the cavity, yield the normalized cavity-antenna detuning $\Delta / \gamma$. Notably, the presence of the glass substrate allows the cavity mode to radiate into the glass at a well-defined angle just beyond the critical angle and, similarly, allows scattering of antenna radiation into the substrate [18]. As the antennas are coherently excited by the cavity field, from which also the cavity radiation originates, the zeroth-order diffraction by the array is expected to overlap with the cavity radiation.

To measure the change of the complex cavity resonance frequency due to the nanoantennas, we compare the frequency and linewidth of the cavity mode with and without the antenna array placed in the near field of the cavity. Our measurement procedure [19] ensures that in both cases the sample is positioned at the same distance to the cavity. Figure 2(a) shows the results for two different arrays of nanoantennas: In the top figure, an antenna array
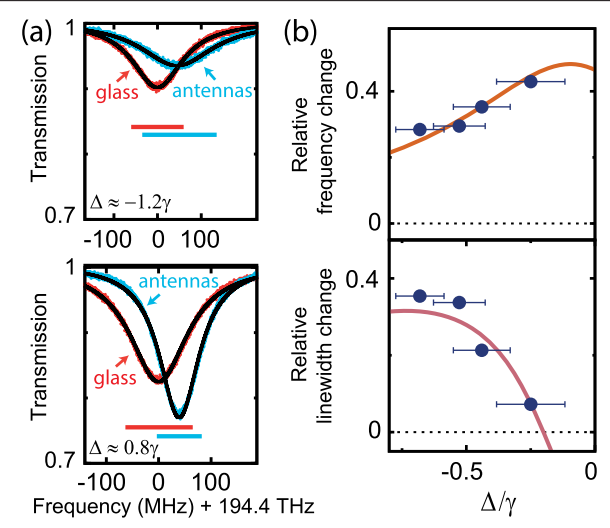

FIG. 2 (color online). (a) Depending on $\Delta$, antenna arrays induce linewidth broadening (top) and narrowing (bottom), while simultaneously inducing the cavity resonance frequency to blueshift. The blue and red horizontal bars represent the linewidths of fitted Lorentzian line shapes (black lines). (b) Shift in resonance frequency and linewidth of the cavity mode, normalized to its linewidth in absence of the antennas [19], due to perturbation by antenna arrays with a constant pitch size and varying antenna length. Top: For all detunings, a blueshift of cavity resonance frequency is observed. Bottom: Approaching $\Delta=0$ induces linewidth narrowing. Both the blueshift and linewidth narrowing are contrary to the expectation based on the particles' polarizability [Fig. 1(a)]. Error bars depict standard deviation, vertical error bars fall within the plot markers. The solid lines represent a coupled mode theory fit.

$[L=360$, pitch 800 (900) $\mathrm{nm}$ along the long (short) axes of the antennas] is seen to induce a broadening of the cavity linewidth, together with a blueshift of the frequency. The latter is clearly surprising, given the fact that $\Delta \approx-1.2 \gamma$ for this sample [19], where one expects a redshift if one only considers the resonant particle's polarizability [Fig. 1(a)]. The bottom panel in Fig. 2(a) shows that for another array $[L=400$, pitch $800(1100) \mathrm{nm}]$ with slightly positive detuning $(\Delta \approx 0.8 \gamma)$ again a blueshift is observed, but this time accompanied by narrowing of the cavity mode. Such a reduction of damping can obviously not be ascribed to the particle's polarizability alone, as its imaginary part is necessarily positive [Fig. 1(a)].

To systematically study these effects, we gradually tune the resonance frequency $\omega_{a}$ by varying the length of the antennas [Fig. 1(f)]. The array pitch is kept fixed at 800 (1500) $\mathrm{nm}$ along the long (short) antenna axes, chosen such that interantenna coupling is negligible. Moreover, in all examples we show, Bragg scattering between clockwise and counterclockwise modes [21] is small enough such that mode splitting induced by the nanoantennas is smaller than the cavity linewidth. Figure 2(b) shows the resulting cavity frequency and linewidth changes, normalized to the cavity linewidth on glass to allow averaging multiple scans [19], as a function of $\Delta / \gamma$. For these negative detunings, a consistent increase of the resonance frequency (i.e., blueshift) is observed that slightly rises as the antenna 
frequency approaches that of the cavity. As such, both the sign and the trend of $\delta \omega_{c}$ are incongruous with the expectation based on the polarizability [Fig. 1(a)]. Moreover, the broadening of the cavity, observed for a detuning of $\Delta \approx-0.6 \gamma$, quickly reduces as the-strongly scattering-antennas are tuned closer to the cavity resonance. We note that these trends are consistently observed also at other periodicities [19] and that the shifts cannot be explained by thermal heating, negligible at the employed powers.

To explore the origin of these surprising results, it is necessary to consider the complete Bethe-Schwinger cavity perturbation equation [10], derived without neglecting radiation:

$$
\begin{aligned}
\delta \omega= & -\omega \frac{\alpha\left|\mathbf{E}_{0}\right|^{2}}{4 U_{0}} \\
& -\frac{i}{4 U_{0}} \int_{\delta V} d A\left[\left(\delta \mathbf{E} \times \mathbf{H}_{0}^{*}\right) \cdot \hat{\mathbf{n}}+\left(\mathbf{E}_{0}^{*} \times \delta \mathbf{H}\right) \cdot \hat{\mathbf{n}}\right] .
\end{aligned}
$$

This full expression, which is also valid in any open, nonHermitian system [19], contains an additional integral term involving radiated fields at a surface $\delta V$ (with normal unit vector $\hat{\mathbf{n}}$ ), enclosing the same volume that was used to evaluate the total energy $U_{0}$. Here, $\delta \mathbf{E}$ and $\delta \mathbf{H}$ are the difference between the fields in the presence $\left(\mathbf{E}_{p}, \mathbf{H}_{p}\right)$ and absence $\left(\mathbf{E}_{0}, \mathbf{H}_{0}\right)$ of the perturbation, and can as such be associated with the field scattered by the perturbation. Thus, the cavity eigenfrequency is additionally modified by an energy flux that is evaluated by combining the fields of the perturbed and unperturbed eigenmodes, i.e., an overlap of scattering by the perturbation and direct cavity radiation. Remarkably, the integral is independent of the distance at which $\delta V$ is chosen, consistent with the fact that it can be associated with radiation of the system. To date, this farfield contribution has been omitted in practically all analyses of cavity perturbation. In a select number of experiments, a reduction of cavity linewidth was observed [22-24] and tentatively attributed to interference of radiation of the cavity and scattering by the perturbation. Importantly, the integral suggests that, in principle, also the resonance frequency (i.e., the real part of $\delta \omega$ ) can be affected by the same mechanism, if the phase difference between cavity and scattered radiation in the far field (captured in $\mathbf{E}_{0}$ and $\delta \mathbf{E}$, respectively) differs from 0 or $\pi$. So far, the frequency has been expected to rely only on the local variation of the applied potential $[3,18,24-27]$ as it is contained in the first term of Eq. (1).

To verify the importance of radiation in a complete description of the induced cavity response, we perform finite-element method eigenfrequency studies with and without antenna [19] in a similar two-dimensional system [Fig. 3(a)], from which we extract the fields $\mathbf{E}_{0}, \mathbf{H}_{0}, \delta \mathbf{E}$, and $\delta \mathbf{H}$. This allows the calculation of the individual terms of Eq. (1), displayed as a function of detuning in Fig. 3(b).
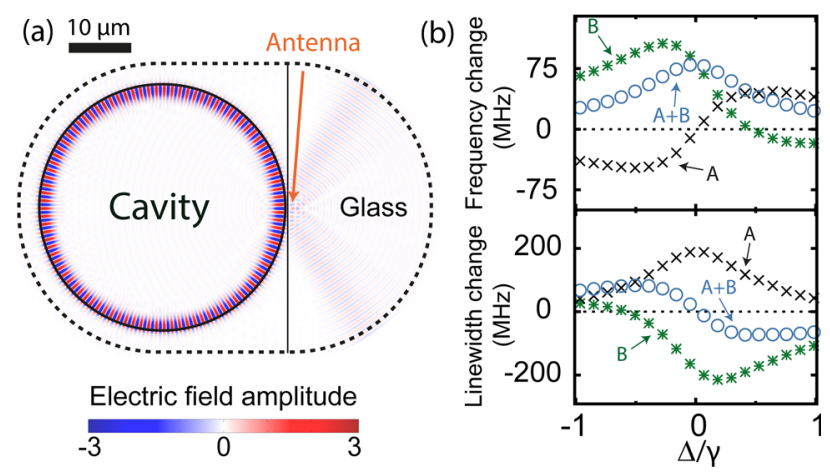

FIG. 3 (color online). (a) Electric field profile in a simulated system containing cavity, antenna and substrate. Both antenna and cavity radiate into the glass under approximately the same angle, resulting in a radiation interaction. The contribution of this interaction is calculated on the dashed black line [integral of Eq. (1)]. The arrow points to the position of the antenna. (b) Top (bottom): the change in resonance frequency (linewidth) calculated using Eq. (1). Black crosses (" $A$ ") show the contribution of the polarizability to the eigenfrequency shift $\delta \omega$, which resembles the line shape as we expect it from a resonant particle [Fig. 1(a)]. The radiative contribution (green stars, " $B$ ") shows distinct blueshifts and linewidth narrowing near $\Delta / \gamma=0$. Adding both terms (blue circles, " $A+B$ ") yields the complete cavity eigenfrequency shift, which matches the experimental trends.

The first term (black crosses, " $A$ "), contributes to $\delta \omega$ according to the expected behavior for a perturbing resonator sketched in Fig. 1(a): it produces a dispersive detuning dependence of $\delta \omega_{\mathrm{c}}$ and a dissipative trend for $\delta \kappa$. This immediately disqualifies this term as an explanation for the measured changes in cavity resonance and linewidth. The second term, i.e., the contribution to the change in eigenfrequency related to the radiation interaction (green stars, " $B$ "), shows a dramatically different behavior: it causes distinct blueshifts and linewidth narrowing around $\Delta / \gamma=0$. Surprisingly, the magnitude of the blueshifts induced by this term can even exceed the contribution due to $\alpha$. This is a key point of our observation, stressing the importance of this new contribution in a model system. The sum of both terms (blue circles, $A+B$ ) yields the complete eigenfrequency shift of the perturbed cavity, which now qualitatively matches our experimental results, blueshifts and linewidth narrowing around $\Delta / \gamma=0$. These calculations thus confirm that radiation interactions in open systems can lead to both cavity blueshifts and linewidth narrowing and importantly, that this effect can even dominate the total change in eigenfrequency of a cavity mode.

It is essential to realize that a modification of the cavity resonance frequency (in contrast to the alteration of the linewidth) due to backaction via the radiation continuum only occurs when the phase $\delta \phi$ between cavity and antenna radiation is different from 0 or $\pi$ [Fig. 4(a)]. The exact strength of this backaction, and as such the sum of direct 


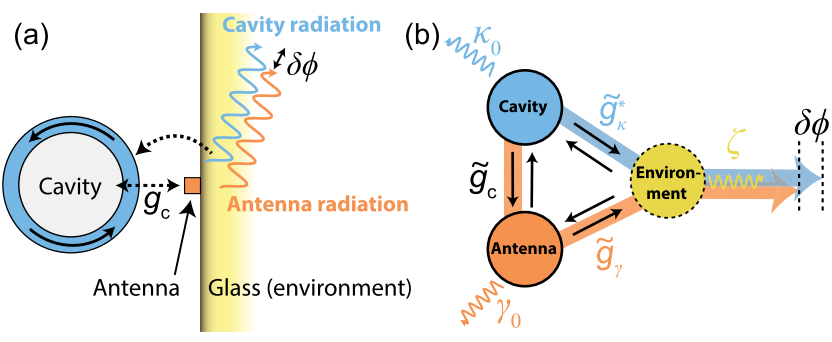

FIG. 4 (color online). (a) The cavity and antenna are coupled with rate $g_{\mathrm{c}}$, which is the near-field interaction scaling with the polarizability of the antenna, and can radiate into the continuum with a certain delay $(\delta \phi)$ with respect to each other. The interference between both radiation profiles and the overlap with the cavity mode results in a backaction effect (single-headed dashed arrow) on the cavity mode. (b) Coupled-mode theory. The cavity and antenna are coupled with rate $\tilde{g}_{\mathrm{c}}$ and couple to the continuum with complex rates $\tilde{g}_{\kappa}=g_{\kappa} e^{\mathrm{i} \phi_{\kappa}}$ and $\tilde{g}_{\gamma}=g_{\gamma} e^{\mathrm{i} \phi_{\gamma}}$, respectively. The difference between $\phi_{\kappa}$ and $\phi_{\gamma}$, summed with the phase response of the antenna, is now equal to the phase difference $\delta \phi . \kappa_{0}$ and $\gamma_{0}$ are the losses of cavity and antenna into noncoupled channels. The environment is modeled as an oscillator with decay rate $\zeta$.

(i.e., due to $\alpha$ ) and indirect (i.e., via the radiation continuum) coupling between cavity and antenna, will depend on the overlap between their radiation profiles and on the value of $\delta \phi$.

This phase difference and its physical connection to backaction can be captured in a simple model [Fig. 4(b)] based on temporal coupled-mode theory [28]. Let us assume for now that the cavity and antenna are coupled with complex coupling rate $\tilde{g}_{\mathrm{c}}=g_{\mathrm{c}} e^{i \phi_{\mathrm{c}}}$ and can both interact with the environment (glass) with coupling rates $\tilde{g}_{\kappa}=g_{\kappa} e^{i \phi_{\kappa}}$ and $\tilde{g}_{\gamma}=g_{\gamma} e^{i \phi_{\gamma}}$, respectively. We treat the joint environment as a separate mode to allow backaction, taking the limit of large decay rate $\zeta$ to mimic a broadband continuum. The cavity and antenna, considered separately, each decay via this continuum at a rate $\kappa_{1}=4\left|\tilde{g}_{\kappa}\right|^{2} / \zeta$ and $\gamma_{1}=4\left|\tilde{g}_{\gamma}\right|^{2} / \zeta$, respectively. This is distinguished from decay into all other, nonoverlapping, modes at rates $\kappa_{0}$ and $\gamma_{0}$, such that $\kappa_{0}+\kappa_{1}=\kappa$ and $\gamma_{0}+\gamma_{1}=\gamma$. Importantly, the phase difference between cavity radiation [blue path, Fig. 4(b)] and antenna scattering [orange path, Fig. 4(b)] in this model now directly relates to $\delta \phi$ via $\delta \phi=\pi+\Phi-\arg [\Delta+\mathrm{i} \gamma / 2]$, where $\Phi=\phi_{\mathrm{c}}+\phi_{\kappa}+\phi_{\gamma}$, and $\arg [\Delta+\mathrm{i} \gamma / 2]$ is the phase response of the antennas [19]. The natural mode of interest, with complex frequency $\omega$, of this hybrid system can be found by equating the determinant of the coupling matrix [19]

$$
M=\left(\begin{array}{ccc}
\omega-\omega_{\mathrm{c}}+\mathrm{i} \kappa_{0} / 2 & \tilde{g}_{\mathrm{c}}^{*} & \tilde{g}_{\kappa} \\
\tilde{g}_{\mathrm{c}} & \Delta+\mathrm{i} \gamma_{0} / 2 & \tilde{g}_{\gamma}^{*} \\
\tilde{g}_{\kappa}^{*} & \tilde{g}_{\gamma} & \mathrm{i} \zeta / 2
\end{array}\right)
$$

to zero. Solving for complex $\omega$ yields

$$
\delta \omega_{\mathrm{c}}-i \delta \kappa / 2=\frac{g_{\mathrm{c}}^{2}-\kappa_{1} \gamma_{1} / 4+i g_{\mathrm{c}} \sqrt{\kappa_{1} \gamma_{1}} \cos \Phi}{\Delta+i \gamma / 2},
$$

which depends on the radiation overlap $\kappa_{1} \gamma_{1}$, (constant) phase $\Phi$, and the coupling rate $g_{\mathrm{c}}$. Because the overlap and phase $\Phi$ cannot be independently chosen, it is impossible to directly fit Eq. (3) to the experimental data. Therefore, we retrieve $\delta \phi$ from the finite element simulation and, using the relation between $\delta \phi$ and $\Phi$, obtain $\Phi=3.76 \times 10^{-4} \pi$. Note that this value of $\Phi$ implies that $\delta \phi \approx \pi / 2$ at $\Delta=0$, completely opposite to the conventionally assumed case where destructive interference $(\delta \phi=\pi)$ only contributes to the linewidth of the cavity mode. Fixing $\Phi$ at this value and constraining the experimentally found values of $\kappa, \Delta$ and $\gamma$ (retrieved from independent spectroscopic measurements, Fig. 1), a fit of Eq. (3) yields excellent correspondence to our data in Fig. 2(b) (solid lines), resulting in an overlap of $\kappa_{1} \gamma_{1} / \kappa \gamma=0.68$ and coupling rate $g_{\mathrm{c}} / 2 \pi=21.6 \mathrm{GHz}$.

Concluding, we have shown how coupling through the radiation continuum leads to cavity blueshifts and linewidth narrowing in a coupled cavity-antenna system. Surprisingly, the nonlocal, radiative effect on the cavity is larger than the induced cavity response due to local perturbations, and is not just an artifact of large radiation losses but can even manifest itself in a high- $Q$ cavity such as studied here. Therefore, similar effects are expected in many other systems where cavity and perturbation can radiate into the same channels, such as photonic crystals [22-24,29] and Fabry-Perot etalons [30-32]. In addition, it was shown that there is a direct link between the phase $\delta \phi$ in the Bethe-Schwinger equation and the contributions to backaction in a coupled-mode model.

Interestingly, these results could also shed new light on radiative interactions in strongly radiating systems such as metamaterials and complex plasmonic resonators. In [14], it was postulated that radiation can be included in the coupling between two resonators by using a complex coupling rate, while in [33], a complex coupling rate was argued to originate from a complex-valued extinction cross section. In fact, such a complex coupling rate between two resonators is easily derived from the coupledmode model presented in this Letter [19]. Furthermore, it will be interesting to see how the concepts and theory established here relate to recently developed methods that rely on the normalization of leaky modes to describe the response of complex photonic systems [34,35]. In a different context, recent advances in optomechanics using simultaneous dispersive and dissipative (i.e., radiative) coupling [36-38] have been reported to enable on resonance cooling [36] and sensitive readout of nanomechanical motion [37]. As such, we expect our work to be directly important to the design of novel optical devices such as 
sensors, for example, and benefit the understanding of the physics of open systems in optics and beyond.

This work is part of the research programme of the Foundation for Fundamental Research on Matter (FOM), which is part of the Netherlands Organisation for Scientic Research (NWO). E. V. gratefully acknowledges support from an NWO Vidi grant. The authors thank Kobus Kuipers for critical reading of the Letter.

*verhagen@amolf.nl

[1] J. M. Raimond, M. Brune, and S. Haroche, Rev. Mod. Phys. 73, 565 (2001).

[2] P. Lodahl, S. Mahmoodian, and S. Stobbe, Rev. Mod. Phys. 87, 347 (2015).

[3] F. Vollmer and S. Arnold, Nat. Methods 5, 591 (2008).

[4] X. Fan, I. M. White, S. I. Shopova, H. Zhu, J. D. Suter, and Y. Sun, Anal. Chim. Acta 620, 8 (2008).

[5] M. Aspelmeyer, T. J. Kippenberg, and F. Marquardt, Rev. Mod. Phys. 86, 1391 (2014).

[6] J. Zhu, S. K. Ozdemir, Y.-F. Xiao, L. Li, L. He, D.-R. Chen, and L. Yang, Nat. Photonics 4, 46 (2010).

[7] J. D. Thompson, T. G. Tiecke, N. P. de Leon, J. Feist, A. V. Akimov, M. Gullans, A. S. Zibrov, V. Vuletić, and M. D. Lukin, Science 340, 1202 (2013).

[8] A. Goban, C.-L. Hung, S.-P. Yu, J. D. Hood, J. A. Muniz, J. H. Lee, M. J. Martin, A. C. McClung, K. S. Choi, D. E. Chang, O. Painter, and H. J. Kimble, Nat. Commun. 5, 3808 (2014).

[9] R. A. Waldron, Proc. IEE Part C Monogr. 107, 272 (1960).

[10] H. A. Bethe and J. Schwinger, Perturbation Theory for Cavities (Massachusetts Institute of Technology, Radiation Laboratory, Cambridge, 1943).

[11] H. Friedrich and D. Wintgen, Phys. Rev. A 32, 3231 (1985).

[12] C. W. Hsu, B. Zhen, J. Lee, S.-L. Chua, S. G. Johnson, J. D. Joannopoulos, and M. Soljačić, Nature (London) 499, 188 (2013).

[13] F. Monticone and A. Alù, Phys. Rev. Lett. 112, 213903 (2014).

[14] R. Taubert, M. Hentschel, J. Kästel, and H. Giessen, Nano Lett. 12, 1367 (2012).

[15] N. Liu, L. Langguth, T. Weiss, J. Kästel, M. Fleischhauer, T. Pfau, and H. Giessen, Nat. Mater. 8, 758 (2009).

[16] D. K. Armani, T. J. Kippenberg, S. M. Spillane, and K. J. Vahala, Nature (London) 421, 925 (2003).

[17] G. Anetsberger, O. Arcizet, Q.P. Unterreithmeier, R. Rivière, A. Schliesser, E. M. Weig, J. P. Kotthaus, and T. J. Kippenberg, Nat. Phys. 5, 909 (2009).

[18] L. Novotny and B. Hecht, Principles of Nano-Optics (Cambridge University Press, Cambridge, 2012).
[19] See Supplemental Material at http://link.aps.org/ supplemental/10.1103/PhysRevLett.115.203904, which includes Ref. [20], for detailed information about our measurement procedure, experimental setup and results for antenna arrays with different pitches, as well as details regarding the finite-element simulation and derivations of Eq. (1)-(3).

[20] F. J. García de Abajo, Rev. Mod. Phys. 79, 1267 (2007).

[21] A. Mazzei, S. Götzinger, L. de S. Menezes, G. Zumofen, O. Benson, and V. Sandoghdar, Phys. Rev. Lett. 99, 173603 (2007).

[22] A. F. Koenderink, M. Kafesaki, B. C. Buchler, and V. Sandoghdar, Phys. Rev. Lett. 95, 153904 (2005).

[23] J. T. Robinson and M. Lipson, Phys. Rev. Lett. 100, 043902 (2008).

[24] M. Burresi, T. Kampfrath, D. van Oosten, J. C. Prangsma, B. S. Song, S. Noda, and L. Kuipers, Phys. Rev. Lett. 105, 123901 (2010).

[25] S. Vignolini, F. Intonti, F. Riboli, L. Balet, L. H. Li, M. Francardi, A. Gerardino, A. Fiore, D. S. Wiersma, and M. Gurioli, Phys. Rev. Lett. 105, 123902 (2010).

[26] L. Lalouat, B. Cluzel, P. Velha, E. Picard, D. Peyrade, J. P. Hugonin, P. Lalanne, E. Hadji, and F. de Fornel, Phys. Rev. B 76, 041102 (2007).

[27] S. Arnold, M. Khoshsima, I. Teraoka, S. Holler, and F. Vollmer, Opt. Lett. 28, 272 (2003).

[28] W. Suh, Z. Wang, and S. Fan, IEEE J. Quantum Electron. 40, 1511 (2004).

[29] M. Barth, S. Schietinger, S. Fischer, J. Becker, N. Nüsse, T. Aichele, B. Löchel, C. Sönnichsen, and O. Benson, Nano Lett. 10, 891 (2010).

[30] A. Mitra, H. Harutyunyan, S. Palomba, and L. Novotny, Opt. Lett. 35, 953 (2010).

[31] D. Chanda, K. Shigeta, T. Truong, E. Lui, A. Mihi, M. Schulmerich, P. V. Braun, R. Bhargava, and J. A. Rogers, Nat. Commun. 2, 479 (2011).

[32] R. Ameling and H. Giessen, Laser Photonics Rev. 7, 141 (2013).

[33] S. Bakhti, N. Destouches, and A. V. Tishchenko, ACS Photonics 2, 246 (2015).

[34] C. Sauvan, J. P. Hugonin, I. S. Maksymov, and P. Lalanne, Phys. Rev. Lett. 110, 237401 (2013).

[35] J. Yang, H. Giessen, and P. Lalanne, Nano Lett. 15, 3439 (2015).

[36] A. Sawadsky, H. Kaufer, R. M. Nia, S. P. Tarabrin, F. Y. Khalili, K. Hammerer, and R. Schnabel, Phys. Rev. Lett. 114, 043601 (2015).

[37] A. C. Hryciw, M. Wu, B. Khanaliloo, and P. E. Barclay, Optica 2, 491 (2015).

[38] A. Xuereb, R. Schnabel, and K. Hammerer, Phys. Rev. Lett. 107, 213604 (2011). 